\title{
The more you look, the more you find: challenging results on FDG-PET CT in a patient with neurofibromatosis type I
}

Patrick Schöffski ${ }^{1,6^{*}}$, Christophe M Deroose ${ }^{2}$, Olivier Gheysens², Nele Lips², Koen Slabbaert ${ }^{3}$, Ben Vancleynenbreugel ${ }^{3}$, Philippe Moerman ${ }^{4}$ and Willy E Peetermans ${ }^{5}$

\begin{abstract}
\section{Background}

FDG-PET/CT is part of the standard diagnostic management of a patients with a large variety of common and less common malignant tumors, based on the increased glucose metabolism within tumors. It is useful for detecting malignant transformation of neurofibromas to MPNST, as malignant MPNST is characterized by higher FDG uptake. A cut-off maximum standardized uptake value $\left(\mathrm{SUV}_{\max }\right)$ of 3.5 discriminates neurofibroma from MPNST (sensitivity: 89\%; specificity 95\%) [1]. High FDG uptake can be seen in non-neoplastic tissues, for instance in inflammatory or infectious lesions. Interpretation of FDG-PET images should thus be done with full knowledge of the case record of the patient.
\end{abstract}

Background: FDG-PET/CT is part of the standard diagnostic management of a patients with a large variety of common and less common malignant tumors, based on the increased glucose metabolism within tumors.

Case presentation: A hybrid fluorodeoxyglucose positron emission tomography and computed tomography (FDG-PET/CT) was performed in a neurofibromatosis patient to rule out relapse of malignant peripheral nerve sheet tumor. The scan revealed non-malignant neurofibromas, a testis seminoma and hypermetabolic syphilitic granulomata.

Conclusion: This case stresses the need to rule out infectious diseases when atypical hypermetabolic lesions are present.

Keywords: Syphilis, PET, Fluorodeoyglucose, Neurofibromatosis, Testicular seminoma

\section{Case presentation}

A 32 year-old homosexual Caucasian male with neurofibromatosis type I (NF1), café-au-lait patches and known superficial and deep neurofibromas consulted us for unexplained fatigue and weight loss. History and

\footnotetext{
*Correspondence: patrick.schoffski@uzleuven.be

1 General Medical Oncology, UZ Leuven, Leuven, Belgium

${ }^{6}$ Department of General Medical Oncology, University Hospitals Leuven, Laboratory of Experimental Oncology, Division of Oncology, Leuven Cancer Institute, Catholic University Leuven, Herestraat 49, Leuven B-3000, Belgium Full list of author information is available at the end of the article
}

biochemistry were unremarkable. His clinical examination revealed known signs of NF1 and a nodular appearance of his right testicle.

FDG-PET/CT was performed to exclude the presence of a NF1-related malignancy (e.g., malignant peripheral nerve sheet tumor, MPNST). It revealed multiple hypermetabolic lesions in the following sites: right scrotum, omentum, peritoneum, neuroforamen $\mathrm{C} 5 / \mathrm{C} 6$, sternum, ribs, both lungs and mediastinum (Figure 1). Based on these findings testicular cancer with abdominal, pulmonary and bone involvement had to be ruled out. Scrotal ultrasound showed a multinodular mass in the right testicle and ipsilateral hypoechogenic inguinal nodules. Serum tumor markers were normal.

The patient underwent a simultaneous orchiectomy and diagnostic laparoscopic resection of inguinal and omental lesions. Histology revealed a $2.6 \mathrm{~cm}$ testicular seminoma with epididymal invasion. The surrounding stroma, funiculus spermaticus and resected omental and inguinal lesions showed a lymphocytic and granulomatous infiltrate, which was initially interpreted as a sarcoid-like reaction or sarcoidosis. There was no evidence for neurofibroma or sarcoma in any of the tissue samples.

A broad laboratory investigation was performed to rule out some common causes of granulomata. Serologies for 


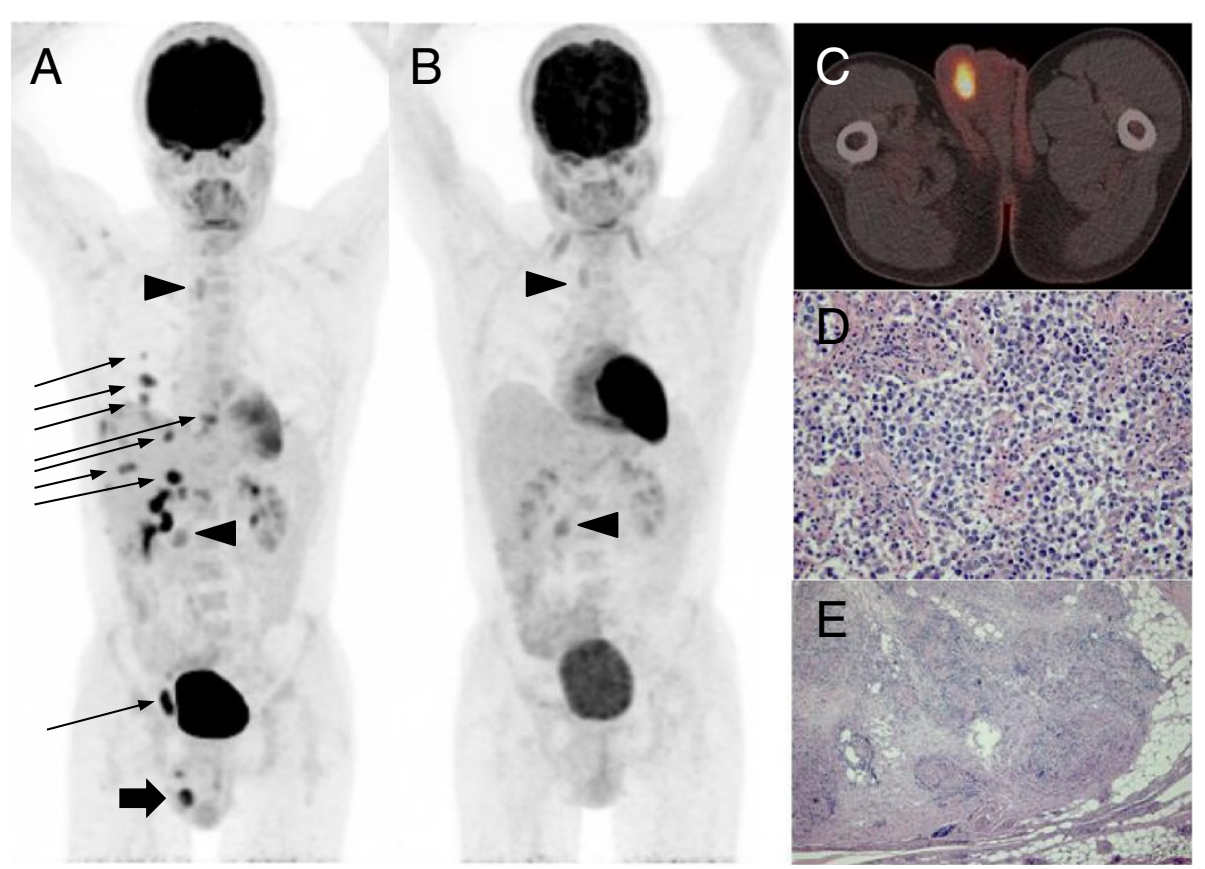

Figure 1 FDG_PET/C T findings. (A) FDG-PET/CT performed at diagnosis (large arrow: hypermetabolic seminoma testis; arrowhead: low FDG-uptake in neurofibroma; small arrow: syphilitic granulomatous lesions); (B) FDG-PET/CT after orchidectomy, diagnostic laparoscopy and two week treatment with doxocycline, showing persistant uptake only in neurofibromas (arrowheads); (C) PET/CT fusion of primary seminoma; (D) hematoxylin/eosin stain of seminoma; and (E) inguinal granuloma.

Bartonella, Brucella, Coxiella, Borellia, Toxoplasma, hepatitis B/C, HIV and HTLV-1 were normal. Tuberculosis was ruled out by specific staining. Serology for Treponema pallidum was positive (Treponema pallidum specific antibody test + , rapid plasma reagin test+, titer $1 / 16$ ). In retrospect, the patient had no history of urogenital complaints or ulcus durum, rash or arthralgia.

We thus interpreted the hypermetabolic granulomatous lesions as active syphilis (primo-infection), for which antibiotic treatment of both the patient (doxycycline $200 \mathrm{mg} /$ day p.o. for 2 weeks because of an IgE-mediated allergy to penicillin) and his serologically-negative partner was initiated. To confirm the working hypothesis of metabolically active syphilis and to rule out distant spread of seminoma, FDG-PET/CT was repeated 21 days after end of therapy, showing complete regression of all hypermetabolic foci, except for known sites of neurofibroma.. We proposed watchful waiting, adjuvant radiotherapy or systemic chemotherapy as options for stage I seminoma, and the patient opted for adjuvant carboplatin AUC 7. He is currently in three-monthly follow-up without clinical, serological or radiological signs of relapse of testicular cancer progression of NF1-related conditions or active syphilis.

FDG-PET/CT is increasingly used as whole body imaging modality in oncology, with high sensitivity and specificity for a number of malignancies. It is useful for detecting malignant transformation of neurofibromas to
MPNST, as malignant MPNST is characterized by higher FDG uptake. A cut-off maximum standardized uptake value $\left(\mathrm{SUV}_{\max }\right)$ of 3.5 discriminates neurofibroma from MPNST (sensitivity: $89 \%$; specificity $95 \%)^{1}$. Seminoma testis is highly FDG-avid (average $\mathrm{SUV}_{\text {mean }}$ 9.2) [2]. FDG$\mathrm{PET} / \mathrm{CT}$ is able to detect seminomatous lesions, is generally not recommended for seminoma staging [3] but is useful for characterization of residual masses after chemotherapy (sensitivity: $80 \%$; specificity $100 \%$ ) [4].

The experience with FDG-PET/CT in syphilis is very limited but hypermetabolic syphilitic lesions in bone, lymph nodes, lung, aorta [5], rectum and brain have been reported only in individual patients. In patients presenting with multiple pathologies quantitative evaluation of the FDG PET images might be of use to ascertain the presence of multiple synchronous diseases. One interesting study [6] proposed a cut off value of $40 \%$ for the difference between two lesions to discriminate metastases from a synchronous secondary primary tumor. When applying this treshold to this patient this suggests that the neurofibromas are not related to the other foci but it does not suggest that the granulomatous lesions are not related to the testis tumor, which is similar to the visual interpretation of the data. Only 3 cases have been described in the literature with a testicular tumor in a NF-1 patient, so occurrence of seminoma in NF1 patients has to be regarded as fortuitous [7-9]. 


\section{Conclusion}

FDG-PET/CT detected an incidental seminoma testis in a NF1-patient with simultaneous hypermetabolic thoracoabdomino-pelvic lesions, which were first attributed to the seminoma, then considered to be sarcoid-like lesions according to histology, and were ultimately diagnosed as syphilitic granulomas based on serology and functional imaging before and after antibiotic treatment. FDG-PET/ $\mathrm{CT}$ cannot differentiate between active inflammation (infectious or not) and malignancy, so metabolic findings always have to be interpreted with caution and in the context of additional clinical information, as shown in our complex case. Infectious diseases should be ruled out in cases with aspecific pathological findings.

\section{Consent}

Written informed consent was obtained from the patient for publication of this Case report and any accompanying images. A copy of the written consent is available for review by the Editor of this journal.

\section{Competing interests}

The authors declare that they have no competing interests.

\section{Authors' contributions}

SP and WEP were the responsible treating physicians of the patient. SP and CMD were responsible for the drafting of the manuscript and creation of the figure. CMD, OG and NL were responsible for the execution and interpretation of the PET scans. KS and BVC performed surgery on the patient. PM was responsible for the pathological diagnoses in the patient. All authors gave critical input to the manuscript and consented with its final version. All authors read and approved the final manuscript.

\section{Acknowledgements}

CMD is a post-doctoral fellow of the Clinical Research Fund of the UZ Leuven.

\section{Author details}

${ }^{1}$ General Medical Oncology, UZ Leuven, Leuven, Belgium. ${ }^{2}$ Nuclear Medicine, UZ Leuven, Leuven, Belgium. ${ }^{3}$ Urology, UZ Leuven, Leuven, Belgium. ${ }^{4}$ Pathology, UZ Leuven, Leuven, Belgium. ${ }^{5}$ General Internal Medicine, UZ Leuven, Leuven, Belgium. ${ }^{6}$ Department of General Medical Oncology, University Hospitals Leuven, Laboratory of Experimental Oncology, Division of Oncology, Leuven Cancer Institute, Catholic University Leuven, Herestraat 49, Leuven B-3000, Belgium.

Received: 17 September 2013 Accepted: 16 May 2014

Published: 29 May 2014

\section{References}

1. Ferner RE, Golding JF, Smith M, Calonje E, Jan W, Sanjayanathan V, O'Doherty M: [18F]2-fluoro-2-deoxy-D-glucose positron emission tomography (FDG PET) as a diagnostic tool for neurofibromatosis 1 (NF1) associated malignant peripheral nerve sheath tumours (MPNSTs): a long-term clinical study. Ann Oncol 2008, 19(2):390-394.

2. Cremerius U, Effert PJ, Adam G, Sabri O, Zimmy M, Wagenknecht G, Jakse G, Buell U: FDG PET for detection and therapy control of metastatic germ cell tumor. J Nucl Med 1998, 39(5):815-822.

3. Krege S, Beyer J, Souchon R, Albers P, Albrecht W, Algaba F, Bamberg M, Bodrogi I, Bokemeyer C, Cavallin-Stahl E, Classen J, Clemm C, Cohn-Cedermark G, Culine S, Daugaard G, De Mulder PH, De Santis M, de Wit M, de Wit R, Derigs HG, Dieckmann KP, Dieing A, Droz JP, Fenner M, Fizazi K, Flechon A, Fossa SD, del Muro XG, Gauler T, Geczi L, et al: European consensus conference on diagnosis and treatment of germ cell cancer: a report of the second meeting of the European
Germ Cell Cancer Consensus group (EGCCCG): part I. Eur Urol 2008, 53:478-496.

4. De Santis M, Becherer A, Bokemeyer C, Stoiber F, Oechsle K, Sellner F, Lang A, Kletter K, Dohmen BM, Dittrich C, Pont J: 2-18fluoro-deoxy-D-glucose positron emission tomography is a reliable predictor for viable tumor in postchemotherapy seminoma: an update of the prospective multicentric SEMPET trial. Journal of clinical oncology : official journal of the American Society of Clinical Oncology 2004, 22:1034-1039.

5. Kosters K, Bleeker-Rovers CP, van Crevel R, Oyen WJ, van der Ven AJ: Aortitis diagnosed by $\mathrm{F}$-18-fluorodeoxyglucose positron emission tomography in a patient with syphilis and HIV coinfection. Infection 2005, 33(5-6):387-389.

6. Dijkman BG, Schuurbiers OC, Vriens D, Looijen-Salamon M, Bussink J, Timmer-Bonte JN, Snoeren MM, Oyen WJ, van der Heijden HF, de Geus-Oei LF: The role of (18)F-FDG PET in the differentiation between lung metastases and synchronous second primary lung tumours. Eur J Nucl Med Mol Imaging 2010, 37(11):2037-2047.

7. Hartley N, Rajesh A, Verma R, Sinha R, Sandrasegaran K: Abdominal manifestations of neurofibromatosis. J Comput Assist Tomogr 2008, 32(1):4-8.

8. Groot-Loonen JJ, Voute PA, de Kraker J: Testicular tumor concomitant with von Recklinghausen's disease. Med Pediatr Oncol 1988, 16(2):116-117.

9. Pasmant E, Vidaud M, Vidaud D, Wolkenstein P: Neurofibromatosis type 1: from genotype to phenotype. J Med Genet 2012, 49(8):483-489.

doi:10.1186/1471-2342-14-19

Cite this article as: Schöffski et al:: The more you look, the more you find: challenging results on FDG-PET CT in a patient with neurofibromatosis type I. BMC Medical Imaging 2014 14:19.

\section{Submit your next manuscript to BioMed Central and take full advantage of:}

- Convenient online submission

- Thorough peer review

- No space constraints or color figure charges

- Immediate publication on acceptance

- Inclusion in PubMed, CAS, Scopus and Google Scholar

- Research which is freely available for redistribution 